\title{
Elastic Property Estimation of Surface Layer of Layered Structure by Measuring Love Wave Propagation Characteristics using Laser Doppler Vibrometer
}

\author{
Yusuke CHIBA*, Tadashi EBIHARA**, Koichi MIZUTANI** and Naoto WAKATSUKI** \\ * Graduate School of Systems and Information Engineering, University of Tsukuba \\ * Faculty of Engineering, Information and Systems, University of Tsukuba \\ E-mail : ebihara@iit.tsukuba.ac.jp
}

\begin{abstract}
The surface properties of a layered structure are useful parameters for coating materials such as functionally graded materials. A Love wave is dispersive, and its propagation characteristics depend on the properties of the material in which in propagates. On this basis, we present an innovative method for measuring the elastic properties of the surface using piezoelectric vibrators and a laser Doppler vibrometer (LDV). Specifically, a Love wave was excited on the surface of a layered structure, and the displacement of the Love wave propagating along the surface layer of the specimen was measured using an LDV. The obtained data were processed in the frequency-wavenumber domain to extract the dispersion curves, and the material properties that minimized the difference between the theoretical and experimental curves were calculated. Experiments were carried out on two materials - poly(methyl methacrylate) and polystyrene and the shear wave velocity and thickness of the surface layer were successfully estimated within a relative error of $10 \%$.
\end{abstract}

Keywords : Love wave, laser Doppler vibrometer, dispersion curve, inverse problem, elastic property

\section{Introduction}

Elastic waves have been widely used for various applications in many engineering fields [1]. In particular, surface acoustic waves (SAWs) and guided waves have been used for the detection of immune or chemical reactions [2-5], the inspection of various structures [6-11], as a special speaker in auditory guides [12, 13], in the development and fabrication of high-performance sensors and manipulators [14-18] and for the measurement of material properties [19-23].

Among these applications, the measurement of layered materials using a guided wave has been studied. For example, Kiełczyński and Szalewski have reported that the material properties of a layered structure specimen (comprising a copper surface layer and steel substrate) can be estimated using a propagating Love wave, which is a type of guided wave [24, 25]. For a Love wave to propagate, the shear wave velocity of the surface layer must be lower than that of the substrate. In this report, the phase velocity of a Love wave (measured at various frequencies) is calculated from the time-of-flight difference of the Love wave, and an optimization problem is solved to find parameters that the phase velocity dispersion curve to converge to experimental values. As a result, the shear modulus, mass density and thickness of the surface layer can be estimated.

In Ref. 25, the generation and observation of a Love wave were performed using a single piezoelectric transducer. Specifically, the piezoelectric transducer was excited to generate a Love wave. The Love wave propagated along the surface layer and was reflected at the edge of the specimen. The reflected wave then back-propagated and was observed at the piezoelectric transducer. Using the measured time-of-flight difference, the phase velocity of the Love wave was calculated. However, this method must consider the effect of wave reflection from all sides of the specimen, which is a source of measurement error.

To solve these problems, we previously proposed a method of measuring Love wave propagation characteristics using a laser Doppler vibrometer (LDV) [26]. In this method, the displacement of a Love wave propagating along a surface layer of a specimen was measured using an LDV with spatial scanning. The spatial frequency of the Love wave was measured by Fourier transform of one-dimensional displacement distribution of the Love wave in time and space domains. Hence, in this method, the propagation characteristics of a Love wave could be measured even in the case of reflection from all sides of the specimen. Moreover, it was not necessary to consider phase unwrapping in this method because the spatial frequency of the Love wave was measured.

As reported in this paper, we designed a system for measuring Love wave propagation using an LDV. Experiments were carried out on two materials - poly(methyl methacrylate) and polystyreneas the surface layer of a layered specimen, and estimation of the elastic properties of the surface layer was performed by solving an inverse problem. This paper consists of five sections. In Sect. 2, we explain the principles of estimating the elastic properties of a surface layer by measuring Love wave propagation on the basis of the propagation characteristics of a Love wave. In Sect. 3, we report experiments carried out on two materials. In this experiment, 


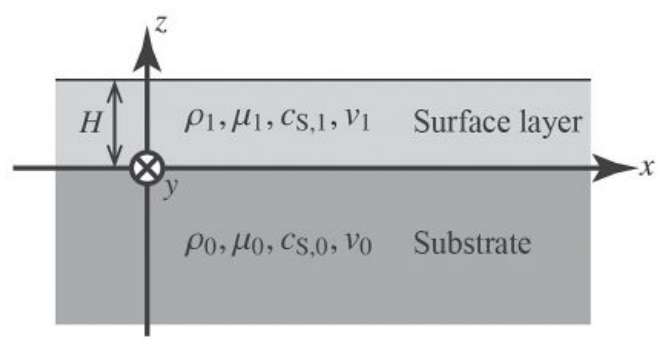

Figure 1 Coordinate system of Love wave propagation between finitethickness elastic surface layer and elastic half-space substrate.

a Love wave was excited on the surface of the layered structure, and the displacement of the Love wave propagating along the surface layer of the specimen was measured using an LDV. The obtained data were processed in the frequency-wavenumber domain to extract the dispersion curves. In Sect. 4, the material properties that minimized the difference between the theoretical and experimental curves are estimated, and the performance of the proposed method is evaluated in terms of estimation accuracy. Section 5 concludes this work.

\section{Measurement principles}

\subsection{Forward problem: propagation characteristics of Love} wave

First, we consider a Love wave propagating in the surface layer on an elastic half-space substrate (Figure 1) [27]. In this figure, variables $\rho, \mu, c_{\mathrm{S}}$ and $v$ denote density, shear modulus, shear wave velocity and displacement in the $y$-direction, respectively. The relation among $\mu, \rho$ and $c_{\mathrm{S}}$ can be expressed as

$$
c_{\mathrm{S}, 0}=\sqrt{\frac{\mu_{0}}{\rho_{0}}} \text { and } c_{\mathrm{S}, 1}=\sqrt{\frac{\mu_{1}}{\rho_{1}}},
$$

where subscripts 0 and 1 indicate the substrate and surface layer, respectively. Variable $H$ indicates the thickness of the layer.

In Figure 1, a shear-horizontal ( $\mathrm{SH}$ ) wave propagates along the $x$-axis with $y$-directional displacement. The wave equations for $\mathrm{SH}$ waves in a surface layer and substrate can be described as

$$
\begin{aligned}
& \frac{\partial^{2} v_{1}}{\partial x^{2}}+\frac{\partial^{2} v_{1}}{\partial z^{2}}=\frac{1}{c_{\mathrm{S}, 1}^{2}} \frac{\partial^{2} v_{1}}{\partial t^{2}} \\
& \frac{\partial^{2} v_{0}}{\partial x^{2}}+\frac{\partial^{2} v_{0}}{\partial z^{2}}=\frac{1}{c_{\mathrm{S}, 0}^{2}} \frac{\partial^{2} v_{0}}{\partial t^{2}}
\end{aligned}
$$

and the boundary conditions can be expressed as

$$
\left\{\begin{array}{c}
v_{1}=v_{0} \\
\mu_{1} \frac{\partial v_{1}}{\partial z}=\mu_{0} \frac{\partial v_{0}}{\partial z} \\
\mu_{1} \frac{\partial v_{1}}{\partial z}=0 \quad(\text { at } z=0),
\end{array}\right.
$$

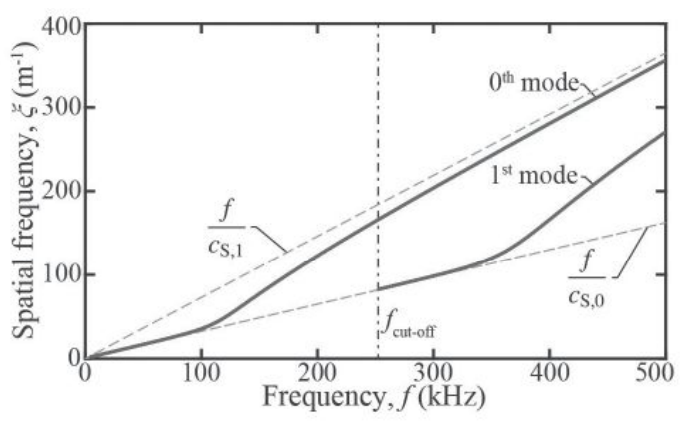

Figure 2 Typical frequency-spatial frequency relations of Love wave obtained from Eq. (9). Blue solid lines indicate dispersion curves of the Love wave. Dashed lines indicate the relationship between the frequency and shear wave velocity in the surface layer and that in the substrate. The material parameters are: $\rho_{1}=$ $1,050 \mathrm{~kg} / \mathrm{m}^{3}, \mu_{1}=1.97 \mathrm{GPa}, c_{\mathrm{s}, 1}=1,370 \mathrm{~m} / \mathrm{s}, H=3 \mathrm{~mm}, \rho_{0}=$ $2,690 \mathrm{~kg} / \mathrm{m}^{3}, \mu_{0}=25.5 \mathrm{GPa}$ and $c_{\mathrm{S}, 0}=3,079 \mathrm{~m} / \mathrm{s}$.

The solutions of eqs. (2) and (3) can be expressed as

$$
\begin{aligned}
& v_{1}=C \cos \left(\sqrt{\left.\frac{c^{2}}{c_{\mathrm{s}, 1}^{2}}-1 k(z-H)\right) \cdot \exp (j k x-j \omega t),}\right. \\
& v_{0}=\mathrm{C} \cos \left(\sqrt{\frac{c^{2}}{c_{\mathrm{s}, 1}^{2}}-1 k H}\right) \cdot \exp \left(\sqrt{1-\frac{c^{2}}{c_{\mathrm{S}, 0}^{2}}} k z\right) \cdot \exp (j k x-j \omega t),
\end{aligned}
$$

where $c, k$ and $\mathrm{C}$ are the phase velocity and wavenumber of the Love wave and an arbitrary constant, respectively. The relation between $c$ and $k$ is expressed as

$$
k=\frac{\omega}{c}=\frac{2 \pi f}{c},
$$

where $\omega$ and $f$ denote the angular frequency and frequency of the Love wave, respectively. Substituting eqs. (6) and (7) into eqs. (4) and (5), and rearranging the equations yield the following characteristic equation of a Love wave propagating between an elastic surface layer and an elastic half-space substrate:

$$
\tan \left(\sqrt{\left.\frac{c^{2}}{c_{\mathrm{S}, 1}^{2}}-1 k H\right)-\frac{\mu_{0} \sqrt{1-c^{2} / c_{\mathrm{S}, 0}^{2}}}{\mu_{1} \sqrt{c^{2} / c_{\mathrm{S}, 1}^{2}-1}}}=0 .\right.
$$

Figure 2 shows the frequency-spatial frequency relations of a Love wave that satisfies eq. (9), where blue solid lines indicate dispersion curves of the Love wave. Here, the spatial frequency, $\xi$, is defined as

$$
\xi=\frac{k}{2 \pi}=\frac{f}{c},
$$

and gray dashed lines indicate the frequency-spatial frequency relations calculated from the shear wave velocity in the substrate, $f / c_{\mathrm{S}, 0}$, and that in the surface layer, $f / c_{\mathrm{S}, 1}$. In Figure 2, the Love wave has multiple dispersion curves; the zeroth mode $(n=0)$ is distributed throughout the entire frequency range, and the first mode $(n=1)$ appears beyond the cutoff frequency, $f_{\text {cutoff }}$, where 


$$
f_{\text {cutoff }}=\frac{n c_{\mathrm{S}, 1}}{2 H \sqrt{1-c_{\mathrm{S}, 1}^{2} / c_{\mathrm{S}, 0}^{2}}}
$$

and $n$ indicates the order of the Love wave propagation mode.

Love wave dispersion curves are determined by the density $\left(\rho_{0}, \rho_{1}\right)$, shear modulus $\left(\mu_{0}, \mu_{1}\right)$, shear wave velocity $\left(c_{\mathrm{S}, 0}, c_{\mathrm{S}, 1}\right)$ and thickness, $H$, of the layer. If the dispersion curves can be obtained experimentally, the material parameters of the surface layer can be estimated by solving the inverse problem.

\subsection{Inverse problem: estimation of elastic properties of layered materials}

In Sect. 2.1, the characteristics of a Love wave propagating between an elastic surface layer and a substrate were described, and the dispersion curves of the Love wave material parameters in the surface layer and substrate were derived. Here, the method of measuring Love wave propagation characteristics is explained.

First, the dispersion curves of a Love wave can be obtained for a Love wave propagating along the $x$-axis: hence, the displacement of a Love wave can be expressed as $v(x, t)$. The complex amplitude spectrum of $v(x, t)$ at an observation point $x$ can be described by a Fourier transform in the time domain as

$$
V(x, f)=\int_{-\infty}^{\infty} v(x, t) \exp (-j 2 \pi f t) d t .
$$

Moreover, the spatial frequency spectrum of $V(x, f)$ can also be expressed by a Fourier transform in the space domain as

$$
\hat{V}(\xi, f)=\int_{-\infty}^{\infty} V(x, f) \exp (j 2 \pi \xi x) d x .
$$

Thus, $\hat{V}(\xi, f)$ has frequency and spatial frequency spectra, and the dispersion curves of a Love wave (as shown in Figure 2) can be obtained by measuring its displacement, $v(x, t)$.

Next, the inverse problem of estimating the material properties of the surface layer is discussed. The objective function of the inverse problem, $Q$, to be minimized is defined as the residual sum of squares between the observed spatial frequency calculated from eq. (13), $\xi_{\text {obs }}(f)$, and the estimated spatial frequency calculated from eq. (9) by using the estimated parameter, $\xi_{\text {est }}(f)$,

$$
Q=\sum_{i=1}^{N}\left(\xi_{\mathrm{obs}}\left(f_{i}\right)-\xi_{\mathrm{est}}\left(f_{i} ; \rho_{1}, c_{\mathrm{S}, 1}, \mu_{1}, H\right)\right)^{2},
$$

where $N$ denotes the number of discrete frequencies, and the arguments of $\xi_{\text {est }}(f), \rho_{1}, c_{\mathrm{S}, 1}, \mu_{1}$ and $H$, are estimated parameters. The objective function is solved as a nonlinear optimization problem by using the Nelder-Mead simplex method embedded in MATLAB $^{\mathbb{B}}[28]$.

\section{Experimental methods and results for elastic property estimation of surface of layered structure}

\subsection{Ultrasonic system for exciting Love wave}

In Sect. 2.2, a method for extracting the dispersion curves from the displacement of a Love wave was explained. In this section, experiments carried out on two synthetic resin materials - poly(methyl methacrylate) (PMMA) and polystyrene (PS) are reported, where the displacement of a Love wave propagating along the surface layer of a specimen was measured using an LDV. The obtained data were processed in the frequency-spatial frequency domain to extract the dispersion curves.
Figure 3 shows our experimental system for measuring the displacement of a Love wave on a layered specimen. The specimen consists of a surface layer $\left[250 \times 40 \times 3\left(\mathrm{~mm}^{3}\right)\right]$ and a substrate $\left[250 \times 40 \times 10\left(\mathrm{~mm}^{3}\right)\right]$, which were adhered using an epoxy-type adhesive. The adhesion layer was much thinner than the surface layer $(<0.1 \mathrm{~mm})$, therefore the specimen was regarded as a twolayered structure in this experimental setup.

In this study, the substrate material was aluminium alloy (A6063) and that of the surface layer was either PMMA or PS. The density, $\rho\left(\mathrm{kg} / \mathrm{m}^{3}\right)$, shear modulus, $\mu(\mathrm{GPa})$, and shear wave velocity, $c_{s}(\mathrm{~m} / \mathrm{s})$, of the aluminium alloy, PMMA and PS are shown in Table 1. Here, the shear wave velocities of the surface layer materials were measured using the method proposed by Imamura and Nakagawa at a nominal frequency of $2.25 \mathrm{MHz}$ [29], and the shear moduli of the surface layer materials were calculated. The density was also calculated by measuring the mass and volume of the materials.

Table 2 shows the experimental conditions for measuring the displacement of the Love wave. The Love wave was excited using two piezoelectric vibrators (C-213, Fuji Ceramics Co.; $3 \times 10 \times 2.5$ $\left.\left(\mathrm{mm}^{3}\right)\right)$ on the specimen, and the resonant frequency was $463 \mathrm{kHz}$ (thickness-shear mode). The poling direction of the vibrators was

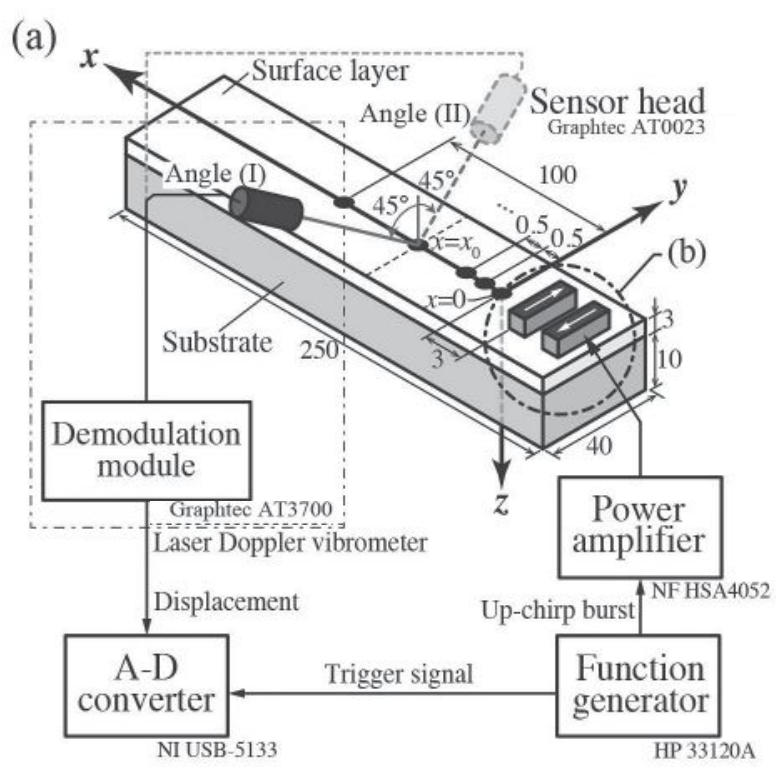

(b)

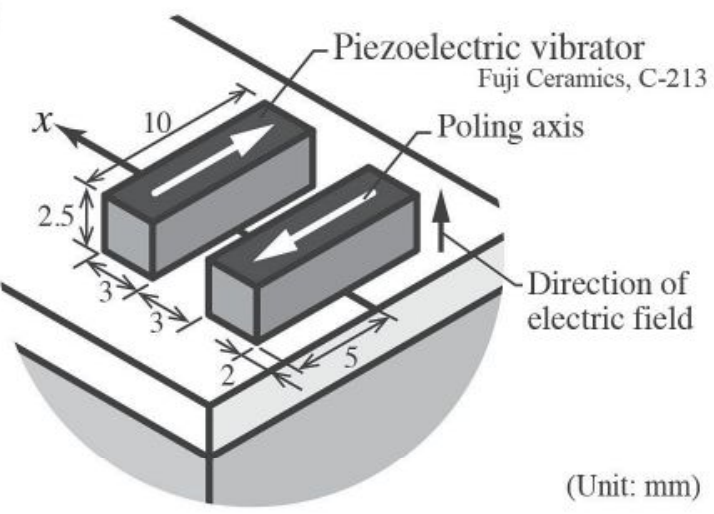

Figure 3 Schematic of experimental setup for measuring displacement of Love wave on surface of layered specimen; (a) measuring system and (b) enlarged view of setup for piezoelectric vibrators. 
Table 1 Material parameters of the specimen. Variables $\rho, \mu$ and $c_{\mathrm{S}}$ denote density, shear modulus and shear wave velocity, respectively.

\begin{tabular}{lrrr}
\hline Materials & $\rho\left(\mathrm{kg} / \mathrm{m}^{3}\right)$ & $\mu(\mathrm{GPa})$ & $c_{\mathrm{S}}(\mathrm{m} / \mathrm{s})$ \\
\hline Substrate: & & & \\
Aluminium alloy (A6063) & 2,690 & 25.5 & 3,079 \\
Surface layer: & & & \\
$\quad$ Poly(methyl methacrylate) & 1,050 & 1.97 & 1,370 \\
Polystyrene & 1,010 & 0.95 & 970 \\
\hline
\end{tabular}

Table 2 Experimental conditions for measuring displacement of Love wave.

\begin{tabular}{|c|c|}
\hline Function generator (FG): HF & P $33120 \mathrm{~A}$ \\
\hline Signal & Linear up-chirp burst \\
\hline Swept frequency & $0-200(\mathrm{kHz})$ \\
\hline Voltage & $1 \mathrm{~V}$ (amplified by up to 100 times) \\
\hline Duration time & $100 \mu \mathrm{s}$ \\
\hline Analog-to-digital converter & (ADC): NI USB-5133 \\
\hline Sampling rate & $2 \mathrm{MHz}$ \\
\hline Recording time & $300 \mu \mathrm{s}$ \\
\hline Bit depth & 8 bit \\
\hline Measurement range & $40 \mathrm{mV}$ (Peak-to-peak value) \\
\hline Laser Doppler vibrometer (I & LDV): GRAPHTEC AT0023/AT3700 \\
\hline Displacement sensitivity & $10^{-7} \mathrm{~m} / \mathrm{V}$ \\
\hline Frequency filter & Band-pass $(0.5-200(\mathrm{kHz}))$ \\
\hline
\end{tabular}
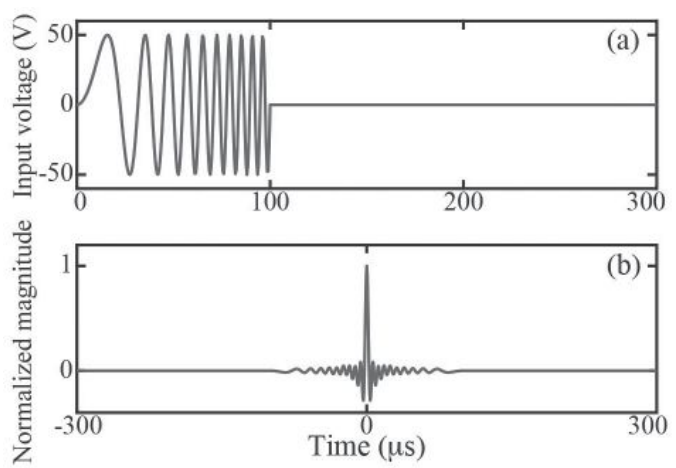

Figure 4 Signal waveform; (a) amplified input signal from function generator through power amplifier and (b) normalized autocorrelation of the input signal.

the $y$-axis, and the vibrators faced each other with an interval of $3 \mathrm{~mm}$. The electric field direction was the $z$-axis. A linear up-chirp burst signal (as shown in Figure 4) was emitted from a function generator (FG; 33120A, Hewlett Packard) in the range of 0-200
$(\mathrm{kHz})$ to avoid the resonant frequency of the piezoelectric vibrator. Therefore, the Love wave propagation characteristics could be measured in a wide range frequency range because vibrators with flat frequency characteristics were used. The input signal was applied to each vibrators through a power amplifier (HSA4052, NF Co.) to amplify it by up to 100 times. Using the above settings, the vibrators were excited.

\subsection{Method of measuring in-plane displacement using laser Doppler vibrometer}

Figure 5 shows the principle for measuring in-plane vibration using an LDV. In general, the laser light of the LDV is radiated toward a plane vibrating orthogonal to the laser light axis, and the vibration of the plane in the normal direction can be detected as the Doppler shift of the laser light [30]. On the other hand, a Love wave has only an SH component, in other words, in-plane vibration, therefore the displacement of a Love wave cannot be measured using a single laser light.

To solve this problem, we devised a means of measuring the displacement of Love wave in-plane vibration using a different optical path of the laser light. In Figure 5(a), the displacement observed using the laser light of an LDV inclined from the $z$-axis by an angle of $\theta$ (counterclockwise as shown in Figure 5(b)), $v_{\mathrm{L}}$, can be described using in-plane and out-of-plane displacement components, $v_{y}$ and $v_{z}$, respectively, as

$$
v_{\mathrm{L}}=v_{y} \sin \theta+v_{z} \cos \theta
$$

Here, the observed displacements at angles of inclination of $\theta$

(a)

(b)
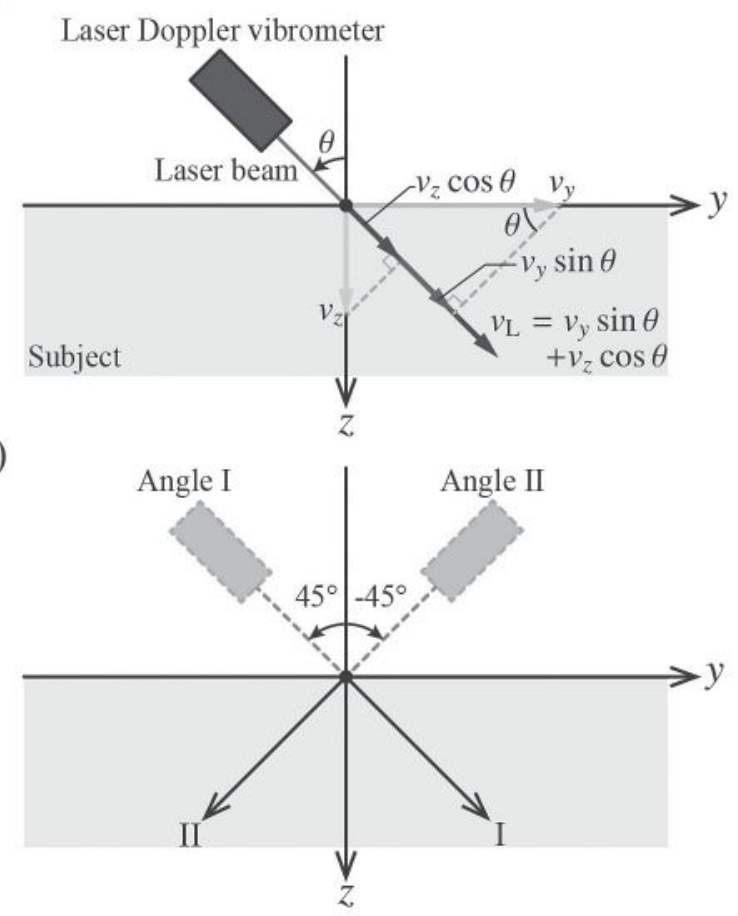

Figure 5 Principle for measurement of in-plane vibrations using LDV in $y$ - $z$ plane; (a) relationship between displacement observed using LDV, $v_{\mathrm{L}}$, and in-plane and out-of-plane displacements, $v_{y}$ and $v_{z}$, respectively, and (b) relationship between $y$ - $z$ plane and I-II coordinate system. 
$=45^{\circ}$ and $\theta=-45^{\circ}, v_{\mathrm{I}}$ and $v_{\mathrm{II}}$, respectively, are expressed as

$$
v_{\mathrm{I}}=\left.v_{\mathrm{L}}\right|_{\theta=45^{\circ}} \text {, and } v_{\mathrm{II}}=\left.v_{\mathrm{L}}\right|_{\theta=-45^{\circ}} \text {. }
$$

Therefore,

$$
\begin{aligned}
{\left[\begin{array}{l}
v_{\mathrm{I}} \\
v_{\mathrm{II}}
\end{array}\right] } & =\left[\begin{array}{cc}
\sin 45^{\circ} & \cos 45^{\circ} \\
\sin \left(-45^{\circ}\right) & \cos \left(-45^{\circ}\right)
\end{array}\right]\left[\begin{array}{l}
v_{y} \\
v_{z}
\end{array}\right], \\
& =\frac{1}{\sqrt{2}}\left[\begin{array}{cc}
1 & 1 \\
-1 & 1
\end{array}\right]\left[\begin{array}{l}
v_{y} \\
v_{z}
\end{array}\right],
\end{aligned}
$$

and, solving the above simultaneous equations yields

$$
\left[\begin{array}{l}
v_{y} \\
v_{z}
\end{array}\right]=\frac{1}{\sqrt{2}}\left[\begin{array}{rr}
1 & -1 \\
1 & 1
\end{array}\right]\left[\begin{array}{l}
v_{\mathrm{I}} \\
v_{\mathrm{II}}
\end{array}\right] .
$$

Here, the in-plane displacement component, $v_{y}$, can be expressed

$$
v_{y}=\frac{v_{\mathrm{I}}-v_{\mathrm{II}}}{\sqrt{2}},
$$

therefore the in-plane displacement can be measured independently.

\subsection{Optical system for measurement of Love wave propagation}

At the point $x=x_{0}$ for the specimen in Figure 3, modulated $\mathrm{He}-\mathrm{Ne}$ laser light was radiated from the sensor head of an LDV(AT0023, Graphtec Co.). As shown in Sect. 3.2, optical measurement using the LDV was performed twice at the same point. First, the sensor head of the LDV was set to angle (I), where the angle between the optical axis of the laser light and the surface of the specimen was $45^{\circ}$. The modulated laser light was scattered on the surface of the specimen and reflected back to the sensor head. The displacement signal was extracted from the scattered HeNe laser light by the demodulation module of the LDV (AT3700, Graphtec Co.). Next, the sensor head of the LDV was set to angle (II), and the displacement was measured. The origin, $x=0$, was at a distance of $3 \mathrm{~mm}$ from the nearer vibrator, and the optical measurement was performed for $100 \mathrm{~mm}$ at $0.5 \mathrm{~mm}$ intervals. Note that all the above measurements were performed on an antivibration table.

The displacement signal was recorded 2,000 times using an analog-to-digital converter (ADC;USB-5133, National Instruments), and an ensemble average was calculated. The recording conditions of the ADC are shown in Table 2. A band-pass filter of $0.5-200(\mathrm{kHz})$ was applied to the displacement signal, and the signal was recorded by the ADC.

\section{Results and discussion}

\subsection{Displacement measurement of Love wave using laser Doppler vibrometer}

Figures 6(a-1) and 6(b-1) show the observed displacements of a Love wave propagating in a layered specimen. The results obtained when the surface layer was PMMA and PS are shown in Figures 6(a-1) and 6(b-1), respectively. The horizontal and vertical axes indicate the elapsed time of the excitation signal of the piezoelectric vibrators emitted from the $\mathrm{FG}$ and the distance from the origin on the surface of the specimen, respectively. In addition, the color scale expresses the displacement of the Love wave obtained from the LDV.

In this experiment, a linear up-chirp burst was applied to the piezoelectric vibrators, and the displacement of the Love wave was measured using the LDV. Therefore, to emphasize the displacement information from the LDV output signal and improve the signalto-noise ratio, pulse compression was performed by calculating the cross-correlation between the input signal (Figure 4(a)) and LDV output signal (Figure 6(a-1) and 6(b-1)). As a result of the pulse compression, the power of the signal shown in Figure 4(a) was concentrated as shown in Figure 4(b). Figures 6(a-2) and
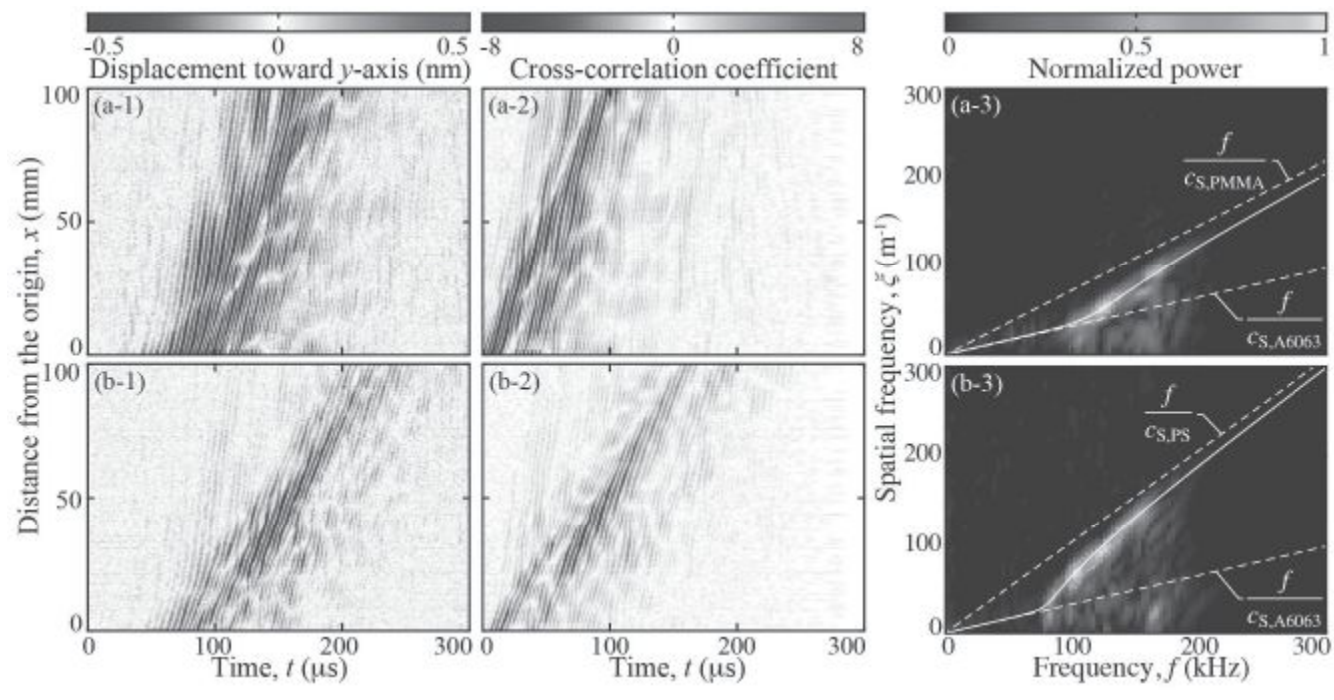

Figure 6 Experimental results in the form of Love wave displacement and dispersion curves for surface layers of (a) PMMA and (b) PS. In these figures, observed and pulse compressed displacements of the Love wave, and frequency-spatial frequency relations of the Love wave calculated using the pulse-compressed displacement are shown as (1), (2) and (3), respectively. 
6(b-2) show the pulse-compressed displacement of the Love wave. By comparing the results of Figure $6(a-1)$ and $6(b-1)$, the displacement information in the LDV output signal was narrowed and emphasized.

The interval of the stripes in Figure 6 became narrower with time because the excitation signal of the piezoelectric vibrator was an up-chirp burst. In addition, stripes with different inclinations were observed. The inclination of the stripes expresses the phase velocity, indicating that a wave having has dispersivity propagated along the layered specimen.

\subsection{Experimental Love wave dispersion curve}

Figures 6(a-3) and 6(b-3) show the experimentally obtained frequency-spatial frequency relations of the Love wave propagating in the layered specimen. The results were obtained by calculating the discrete Fourier transform of Figures 6(a-2) and 6(b-2). The horizontal and vertical axes indicate the frequency, $f$, and spatial frequency, $\xi$, of the Love wave, respectively. Moreover, the color scale expresses the normalized power. In Figures $6(a-3)$ and $6(b-$ 3 ), solid lines indicate the dispersion curves of the Love wave obtained using the measured value calculated from eq. (9). Dashed lines indicate the relationship between the frequency and spatial frequency calculated from the shear wave velocity of the surface layer, $f / c_{\mathrm{S}, \mathrm{PMMA}}$, and that of the substrate, $f / c_{\mathrm{S}, \mathrm{PS}}$.

From Figures 6(a-3) and 6(b-3), both the dispersion curves of the Love wave were in good agreement. Comparing Figures 6(a-3) and 6(b-3), the inclination of the observed dispersion curve in Figure $6(b-3)$ is greater than that in Figure 6(a-3). Here, the shear wave velocity of PS $\left(c_{\mathrm{S}, \mathrm{PS}}=970 \mathrm{~m} / \mathrm{s}\right)$ is smaller than that of PMMA $\left(c_{\mathrm{S}}\right.$, ${ }_{\text {PMMA }}=1,370 \mathrm{~m} / \mathrm{s}$ ). If the thickness of the surface layer is the same, the inclination of the Love wave dispersion curve is greater when the surface layer has a lower shear wave velocity. Thus, the elastic property can be evaluated qualitatively from the shape of the Love wave dispersion curve.

\subsection{Results of estimating material properties of surface layer by solving inverse problem}

Table 3 shows the estimated parameters of the surface layer. These estimated parameters minimize eq. (14) and are the average values of 30 optimization trials. The initial parameters were set to random values within a range of $\pm 50 \%$ of the values in Table 1 .

First, the optimization problem was solved for three parameters: $\left(\rho_{1}, c_{\mathrm{S}, 1}, H\right)$ and $\left(\rho_{1}, \mu_{1}, H\right)$. The estimation results and relative error rates obtained using $\left(\rho_{1}, c_{\mathrm{S}, 1}, H\right)$ and $\left(\rho_{1}, \mu_{1}, H\right)$ are shown in rows 5-6 and rows 7-8 of Table 3 , respectively. Although the relative error rate of the estimated parameters in the case of PS, $\mu_{1}, c_{\mathrm{S}, 1}$ and $H$, were within $10 \%$, the remaining parameters were estimated with an error rate of over $10 \%$.

Next, the optimization problem was solved using two estimated parameters excluding the density $\rho_{1}:\left(c_{\mathrm{S}, 1}, H\right)$ and $\left(\mu_{1}, H\right)$. The estimation results and relative error rates obtained using $\left(c_{\mathrm{S}, 1}, H\right)$ and $\left(\mu_{1}, H\right)$ are also shown in rows 9-10 and rows 11-12 of Table 3 , respectively. For both materials, the relative error rates of the parameters were smaller than those in the case of three estimated parameters because the global solution tended to converge with decreasing dimension of the objective function. In particular, two of the parameters among $\mu_{1}, \rho_{1}$ and $c_{\mathrm{S}, 1}$ could not be estimated simultaneously, as shown by eq. (1), therefore it was difficult to demonstrate that the optimization problem was solved using three estimated parameters.
Table 3 Estimated material parameters of surface layer. The estimated parameters are averages of 30 optimization trials. When the estimated parameters are $\left(c_{\mathrm{S}, 1}, H\right)$ and $\left(\mu_{1}, H\right)$, the density $\rho_{1}$ is a known parameter obtained by measurement. The relative error rate is the ratio of the estimated parameters to the measured values.

\begin{tabular}{|c|c|c|c|c|c|c|c|c|}
\hline & \multicolumn{4}{|c|}{ (a) Poly(methly methacrylate) } & \multicolumn{4}{|c|}{ (b) Polystyrene } \\
\hline & $\rho_{1}$ & $\mu_{1}$ & $c_{\$, 1}$ & $H$ & $\rho_{1}$ & $\mu_{1}$ & $c_{8,1}$ & $H$ \\
\hline & $\left(\mathrm{kg} / \mathrm{m}^{3}\right)$ & $(\mathrm{GPa})$ & $(\mathrm{m} / \mathrm{s})$ & (mm) & $\left(\mathrm{kg} / \mathrm{m}^{3}\right)$ & (GPa) & $(\mathrm{m} / \mathrm{s})$ & (mm) \\
\hline Measured values & 1,050 & 1.97 & 1,370 & 3.1 & 1,010 & 0.95 & 970 & 3.0 \\
\hline Estimated parameters $\left(\rho_{1}, c_{5,1}, H\right)$ & 132 & - & 1.659 & 4.39 & 601 & - & 1,002 & 3.05 \\
\hline Relative error rate $(\Re)\left(\rho_{1}, c_{\mathrm{s}, 1}, H\right)$ & -87.4 & - & +21.1 & +41.6 & -40.5 & - & +3.27 & +1.52 \\
\hline Estimated parameters $\left(\rho_{1}, \mu_{1}, H\right)$ & 382 & 0.88 & - & 3.94 & 878 & 0.86 & - & 2.97 \\
\hline Relative error rate $(\Im)\left(\rho_{1}, \mu_{1}, H\right)$ & .63 .7 & -55.3 & - & +27.2 & -13.1 & -9.71 & - & -0.99 \\
\hline Estimated parameters $\left(c_{5,1}, H\right)$ & - & - & 1,456 & 3.11 & - & - & 988 & 2.92 \\
\hline Relative error rate $(\mathscr{F})\left(c_{\mathrm{S}, 1,}, H\right)$ & - & - & +6.31 & +0.29 & - & - & +1.90 & -2.57 \\
\hline Estimated parameters $\left(\mu_{1}, H\right)$ & - & 2.22 & - & 3.11 & - & 0.99 & - & 2.92 \\
\hline Relative error rate $(\%)\left(\mu_{1}, H\right)$ & - & +13.1 & - & +0.33 & - & +3.85 & - & $-2,60$ \\
\hline
\end{tabular}

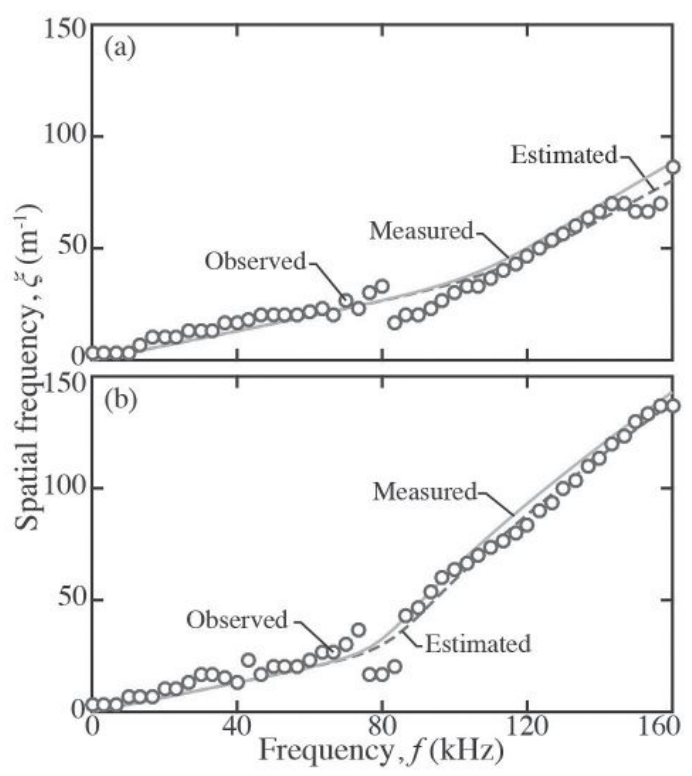

Figure 7 Estimated spatial frequency of Love wave (parameters: $c_{\mathrm{S}, 1}$ and H); (a) PMMA and (b) PS. Blue circles denote observed spatial frequencies, and orange solid and red dashed lines indicate the measured and estimated spatial frequency dispersion curves of the Love wave calculated from eq. (9), respectively.

When the optimization problem was solved using two estimated parameters, the density, $\rho_{1}$, was treated as a known parameter. This is because the purpose of this paper is to estimate the elastic property of a surface layer material. In the case of the estimated parameters $\left(c_{\mathrm{S}, 1}, H\right)$, the relative error rate was smaller than that for the estimated parameters $\left(\mu_{1}, H\right)$. In this study, the shear wave velocity and density of each surface layer material were measured, as mentioned in Sect. 3.1, therefore it was considered that the shear modulus contained the measurement error of the shear wave velocity and density. One of our future tasks is to improve the measurement accuracy of $c_{\mathrm{S}, 1}$ since it determines the estimation accuracy of the parameters. 
Figure 7 shows the spatial frequency dispersion curve obtained from the estimated parameters $\left(c_{\mathrm{S}, 1}, H\right)$. The horizontal and vertical axes indicate the frequency and spatial frequency of the Love wave, respectively. Blue circles denote observed spatial frequencies, and orange solid and red dashed lines indicate measured and estimated spatial frequency dispersion curves of the Love wave calculated from eq. (9), respectively. To estimate the spatial frequency dispersion curves, the observed spatial frequency data with a sufficient gain [0-163 (kHz)] were utilized [Figures 6(a-3)(b-3)], resulting in $N=49$ data points with frequency resolution $\Delta$ $f=3.333 \mathrm{kHz}$. For each material, the spatial frequency dispersion curves calculated using the estimated parameters $\left(c_{\mathrm{S}, 1}, H\right)$ agreed with those calculated using measured values. In conclusion, it was confirmed that the proposed method can estimate the elasticity and thickness of the surface layer independently. Moreover, the above results indicate that the shear wave velocity, $c_{\mathrm{S}, 1}$, is suitable for use as an estimated parameter rather than the shear modulus, $\mu_{1}$.

\section{Conclusions}

In this study, we designed a system for measuring Love wave propagation using a laser Doppler vibrometer and estimating the elastic properties of two surface layer materials -poly(methyl methacrylate) and polystyrene. It was found that the shear wave velocity, $c_{\mathrm{S}, 1}$, and thickness, $H$, of the surface layer, were successfully estimated within a relative error of $10 \%$.

When the inverse problem was solved using $\left(c_{\mathrm{S}, 1}, H\right)$ to estimate the material properties, $\rho_{1}$ was treated as a known parameter. Therefore, the proposed method is suitable for estimation of the shear elasticity or thickness of a surface layer. In addition, we consider that the proposed method can be applied to material whose density deviation is small. For example, it is expected that shear elasticity and thickness of coating materials will be suitable for the measurement of material properties because the change in the elasticity with time is larger than that of the density.

\section{References}

[1] Mizutani, K., Wakatsuki, N., Ebihara, T.; "Introduction of measurement techniques in ultrasonic electronics: Basic principles and recent trends," Jpn. J. Appl. Phys. 55, 07KA02 (16 pages) (2016).

[2] Jiang, Y., Tan, C. Y., Tan, S.Y., Wong, M. S. F., Chen, Y. F., Zhang, L., Yao, K., Gan, S. K. E., Verma, C., 5 Tan, Y.-J.; "SAW sensor for influenza A virus detection enabled with efficient surface fictionalization," Sens. Actuator B 209, 7884 (2015).

[3] Goto, M., Yatsuda, H., Kondoh, J.; "Effect of viscoelastic film for shear horizontal surface acoustic wave on quartz," Jpn. J. Appl. Phys. 54, 07HD02 (5 pages) (2015).

[4] Yang, J.-P., Shen, C.-Y., Chen, Y.-J., Huang, H.-C., Hwang, R.-C.; "The estimations of ammonia concentration by using neural network SH-SAW sensors," Expert Syst. Appl. 38, 4774-4779 (2011).

[5] Kogai, T., Yoshimura, N., Mori, T., Yatsuda, H.; "LiquidPhase Shear Horizontal Surface Acoustic Wave Immunosensor," Jpn. J. Appl. Phys. 49, 07HD15 (4 pages) (2010).

[6] Hosaka, Y., Imano, K.; "Sensitive tint visualization of A2 mode Lamb waves," J. Soc. Mater. Eng. Resour. Jpn. 28, 1924 (2017).

[7] Hosaka, Y., Imano, K.; "Sensitive tint visualization system for Lamb waves propagation in a glass plate," Acoust. Sci.
Tech. 38, 165-167 (2017).

[8] Zhang, J., Ma, H., Yan, W., Li, Z.; "Defect detection and location in switch rails by acoustic emission and Lamb wave analysis: A feasibility study," Appl. Acoust. 105, 67-74 (2016).

[9] Loveday, P. W., Long, C. S.; "Laser vibrometer measurement of guided wave modes in rail track," Ultrason. 57, 209-217 (2015).

[10] Ouchi, A., Sugawara, A., Ohara, Y., Yamanaka, K.; "Subharmonic phased array for crack evaluation using surface acoustic wave," Jpn. J. Appl. Phys. 54, 07HC05 (6 pages) (2015).

[11] Imano, K., Endo, T.; "Experimental Study on the Mode Conversion of Lamb Wave Using a Metal Plate Having a Notch Type Defect," Int. J. Soc. Mater. Eng. Resour. 19, 2023 (2013).

[12] Fujii, A., Wakatsuki, N., Mizutani, K.; "Analysis of propagation characteristics of flexural wave in honeycomb sandwich panel and design of loudspeaker for radiating inclined sound," Jpn. J. Appl. Phys. 54, 07 HB08 (8 pages) (2015).

[13] Fujii, A., Wakatsuki, N., Mizutani, K.; “A planar acoustic transducer for near field acoustic communication using evanescent wave," Jpn. J. Appl. Phys. 53, 07KB07 (6 pages) (2014).

[14] Kakio, S., Hosaka, K.; "Loss reduction of leaky surface acoustic wave by loading with high-velocity thin film, "Jpn. J. Appl. Phys. 55, 07KD11 (6 pages) (2016).

[15] Tang, G., Han, T., Chen, J., Zhang, B., Omori, T., Hashimoto, K.; "Thin plate model for transverse mode analysis of surface acoustic wave devices," Jpn. J. Appl. Phys. 55, 07KD09 (5 pages) (2016).

[16] Oishi, M., Hamashima, H., Kondoh, J.; "Measurement of cantilever vibration using impedance-loaded surface acoustic wave sensor," Jpn. J. Appl. Phys. 55, 07KD06 (6 pages) (2016).

[17] Fukaya, T., Kondoh, J.; "Experimental consideration of droplet manipulation mechanism using surface acoustic wave," Jpn. J. Appl. Phys. 54, 07HE06 (5 pages) (2015).

[18] Kakio, S., Fukasawa, H., Hosaka, K.; "Love-type surface

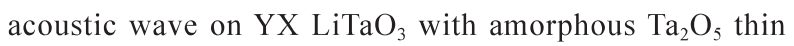
film," Jpn. J. Appl. Phys. 54, 07 HD03 (4 pages) (2015).

[19] Tsuji, T., Oizumi, T., Takeda, N., Akao, S., Tsukahara, Y., Yamanaka, K.; "Temperature compensation of ball surface acoustic wave sensor by two-frequency measurement using undersampling," Jpn. J. Appl. Phys. 54 07HD13 (6 pages) (2015)

[20] Xiao, X., Shan, X. M., Kayaba, Y., Kohmura, K., Tanaka, H., Kikkawa, T.; "Young's modulus evaluation by SAWs for porous silica low-k film with cesium doping," Microelectron. Eng. 88, 666-670 (2011).

[21] Cegla, F. B., Cawley, P., Lowe, M. J. S.; "Material property measurement using the quasi-Scholte mode-A waveguide sensor," J. Acoust. Soc. Am. 117, 1098-1107 (2005).

[22] Morita, T., Sugimoto, M., Kondoh, J.; "Measurements of Standard-Viscosity Liquids Using Shear Horizontal Surface Acoustic Wave Sensors," Jpn. J. Appl. Phys. 48, 07GG15 (4 pages) (2009).

[23] Kondoh, J., Hayashi, S., Shiokawa, S.; "Simultaneous Detection of Density and Viscosity Using Surface Acoustic Wave Sensors," Jpn. J. Appl. Phys. 40, 3713-3717 (2001). 
[24] Kiełczyński, P., Szalewski, M.; "Determination of the elastic properties of thin layers and graded materials using generalized Love waves," IEICE Tech Rep. 109, 117-122 (2010).

[25] Kiełczyński, P., Szalewski, M.; "Inverse determination of thickness and elastic properties of thin layers and graded materials using generalized Love waves," Proc. 2010 IEEE Int.Ultrason. Symp., 2235-2238 (2010).

[26] Chiba, Y., Ebihara, T., Mizutani, K., Wakatsuki, N.; "Measurement of Love wave propagation characteristics along elastic substrate with a viscoelastic surface layer,"
Proc. Symp. Ultrason. Electron. 37, 2P2-2 (2 pages) (2016).

[27] Love, A. E. H.; Some problems of geodynamics (Cambridge University Press, Cambridge, 1911) p.162.

[28] Lagarias, J. C., Reeds, J. A., Wright, M. H., Wright, P. E.; "Convergence properties of the NelderMead simplex method in low dimensions," SIAM J. Optim. 9, 112-147 (1998).

[29] Imamura, T., Nakagawa, Y.; Japan Patent 3733429 (2006).

[30] Morikawa, R., Nakamura, K., Ueha, S.; "Measuring the structural intensity by sensing the in-plane vibration," $J$. Acoust. Soc. Jpn. (E) 18, 201-203 (1997). 